\title{
Steep magnetic trap for ultra cold atoms
}

\author{
V. Vuletic, T. W. HÄnsch and C. Zimmermann \\ Sektion Physik, Ludwig-Maximilians-Universität \\ Schellingstraße 4, 80799 München, Germany \\ Max-Planck-Institut für Quantenoptik \\ Postfach 1513, 85740 Garching, Germany
}

(received 13 June 1996; accepted in final form 24 September 1996)

PACS. 32.80Pj - Optical cooling of atoms; trapping.

PACS. 42.50-p - Quantum optics.

\begin{abstract}
A combination of current coils and ferromagnetic pole shoes is used to construct a magnetic quadrupole trap for ultra cold neutral atoms. Field gradients of up to $3000 \mathrm{G} / \mathrm{cm}$ have been achieved continuously with an electric power consumption of only 10 watts. A maximum field gradient of $1 \mathrm{~T} / \mathrm{cm}$ is calculated for a current that can be applied for several seconds. The trap has been loaded with $8 \times 10^{6}$ lithium atoms $\left({ }^{7} \mathrm{Li}\right)$ from a magneto-optical trap and the energy distribution determined by stepwise reduction of the trapping potential. Finally, magnetic trapping of fermionic ${ }^{6} \mathrm{Li}$ has been demonstrated.
\end{abstract}

Recently, the technique of evaporative cooling has been successfully used to cool magnetically trapped alkali atoms into the regime of quantum degeneracy. This spectacular breakthrough opens a rich field for new experiments, amongst which Bose-Einstein condensation as observed last year is only a first example [1]-[3]. Evaporative cooling, first demonstrated with hydrogen ten years ago [4], has turned out to be very efficient also for alkali atoms [5], [6] and will probably remain the key technique for future studies. As a basic condition, it requires the thermalization of the external degrees of freedom in a gas. Magnetically trapped atoms typically thermalize via binary elastic collisions which for low temperatures are dominated by $s$-wave scattering. Thus, the $s$-wave scattering length is a critical parameter which, in connection with the atomic density, determines the collision rate. For a given lifetime of the atoms inside the trap an improved collision rate allows for a more efficient cooling trajectory. Atoms such as rubidium and sodium with their comparably large scattering lengths are therefore favored candidates. However, from a less technical point of view, lithium is an even more interesting atom since it is the only alkali with stable bosonic and fermionic isotopes. Lithium is thus particularly well suited to study the impact of spin statistics on a degenerate quantum gas. Unfortunately, its elastic scattering cross-section is 13 times smaller than that of sodium and evaporative cooling is far more difficult [7]. Experiments with trapped bosonic lithium have been carried out in a static Ioffe trap made from permanent magnets [3] and evaporative cooling has been demonstrated, however, with a long cooling time of about 10 min. Faster cooling can be achieved by adiabatic compression of the trapped gas, thus increasing the density and the collision rate. This, however, requires very steep magnetic traps with variable field 


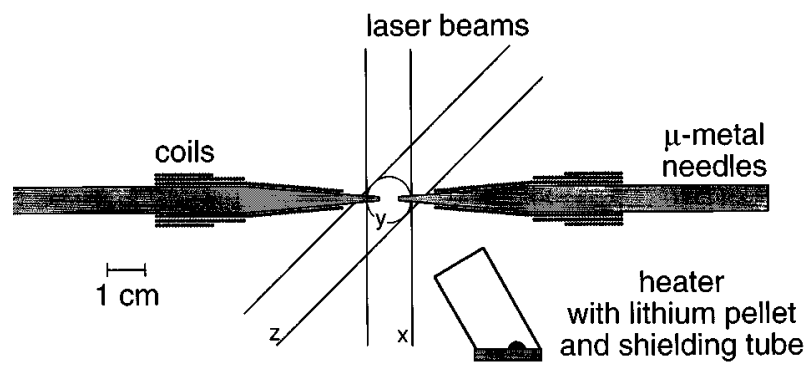

Fig. 1. - Design of a steep magnetic quadrupole trap. A pair of ferromagnetic needles enhances, collects and focuses the magnetic field resulting in a high gradient between the needles.

gradients. High gradients can be produced with microscopic arrangements [8] and by employing ferromagnetic material. With the trap design described in this article continuous field gradients of $3000 \mathrm{G} / \mathrm{cm}$ have been achieved with only 10 watts of electrical-power consumption. The high gradients are due to ferromagnetic needles that collect and focus the magnetic fields of two coils with opposite currents (fig. 1). If operated in a pulsed mode with three times larger currents $(15 \mathrm{~A})$, the heat capacity of the coils will allow for gradients of $1 \mathrm{~T} / \mathrm{cm}$ for at least $7 \mathrm{~s}$. In such a tight potential trap ("tip trap") a gas of lithium atoms is expected to thermalize within several ten milliseconds at densities and temperatures as can be achieved when loading from a magneto-optical trap.

The geometry of the tip trap is sketched in fig. 1 . The needles, which are spaced by $5.5 \mathrm{~mm}$, consist of a ferromagnetic metal that has been selected for its small remanence (mu-Metal, hart; Vakuumschmelze Hanau, saturation field strength $B_{\text {sat }}=0.8 \mathrm{~T}$, relative magnetic permeability $\mu=10000$ ). When the current is rapidly turned off within $200 \mu \mathrm{s}$, the magnetic field drops to $30 \%$ and then falls off with the longer time constant $(800 \mu \mathrm{s})$ of the ferromagnetic material. From the measured residual field of a single tip $(0.5 \mathrm{G}$ after the tip had been magnetized to produce $350 \mathrm{G}$ at the same position) the residual gradient at the trap center is calculated to be $1.5 \mathrm{G} / \mathrm{cm}$ after a gradient of $1000 \mathrm{G} / \mathrm{cm}$ has been applied. The coils are made of capton-isolated copper wire (0.6 mm diameter) which is wrapped in three layers directly onto the needles (150 turns per coil). The two electromagnets are mounted onto a copper frame and placed inside a vacuum chamber that is evacuated to $10^{-10} \mathrm{mbar}$. We did not observe any degradation of the vacuum due to the trap arrangement up to continuous currents of $2 \mathrm{~A}$. The dimensions and the geometry of the coils and the needles have been optimized by means of a commercial computer program that calculates the magnetic field for a given cylindrical symmetric distribution of coils and ferromagnets. Figure 2 shows the calculated magnetic-field modulus. If operated at a current of $1 \mathrm{~A}$ the field gradients in the region between the tips amount to $710 \mathrm{G} / \mathrm{cm}$ in the axial and $350 \mathrm{G} / \mathrm{cm}$ in the radial direction, with a trap depth of $52 \mathrm{G}$. Larger gradients are possible for smaller separation of the two needles. However, the number of atoms in a magneto-optical trap (MOT) operated between the two tips is substantially reduced. In fact, the tip distance turns out to be a critical parameter and at a separation of $4 \mathrm{~mm}$ a 20 -fold reduction in atom number has been observed. Note that in the radial direction at distances from the center larger than $2 \mathrm{~mm}$ the magnetic gradient rolls off and the field forms a fairly constant plateau around $50 \mathrm{G}$.

We operate the tip trap as a lithium MOT by reducing the current to $120 \mathrm{~mA}$ corresponding to an axial field gradient of $90 \mathrm{G} / \mathrm{cm}$. Two light beams $(8 \mathrm{~mm}$ diameter) with standard MOT polarizations are propagating perpendicularly to the symmetry axis of the trap, while a third beam passes the trap at an angle of about $45^{\circ}$ (in the following called the $z$-direction in contrast 
to the axial direction which defines the symmetry axis of the trap). The laser light near $671.962 \mathrm{~nm}\left(\mathrm{D}_{2}\right.$ resonance) is produced by a titanium-sapphire laser, and the two frequency components for trapping and repumping are realized by the lower and the upper sideband of an electro-optical phase modulator operated near $405 \mathrm{MHz}$. Each sideband contains about $30 \%$ of the total laser power and the light intensity at the trap amounts to $200 \mathrm{~mW} / \mathrm{cm}^{2}$ per hyperfine transition frequency component. The trap is loaded from a ceramic, UHV compatible heater element that is placed close to the MOT $(5 \mathrm{~cm})$ and evaporates atoms from a small piece of lithium metal. The laser frequency is controlled by means of a beat signal with a laser diode that is locked to a reference resonance of the Doppler-free $\mathrm{D}_{2}$-line, observed by frequency modulation spectroscopy and containing the unresolved transitions from the $F=2$ ground state to the excited hyperfine states $F^{\prime}=3,2,1$. The experiments described in this article are carried out with $-40 \mathrm{MHz}$ detuning of the trapping sideband relative to the reference laser frequency.

We find that the same number of atoms can be collected with linear polarization of the two counterpropagating laser beams in the $z$-direction (see fig. 1). However, with circularly polarized light of a standard MOT configuration the alignment of the laser beams is less critical. We assume that in the case of linear polarization the axial confinement of the atoms is achieved by spatially misbalanced light intensities of the two counterpropagating laser beams in the $z$-direction [9]. The inhomogeneity may be caused by a slight mutual angle misalignment between the two beams. Atoms approaching one of the tips will then enter the counterpropagating beam while leaving the copropagating beam, and the resulting radiation pressure misbalance acts as a restoring force. This assumption is supported by the observation that the cloud inside the MOT is elongated in the axial direction, while for a conventional MOT one would expect this to be the short axis. The temperature measurement described below is carried out with linear polarization of the $z$-beam, while for the following purely magnetic trapping experiments circular polarization is used.

The atom number has been estimated by monitoring the fluorescence from the MOT with a calibrated photodiode. From the measured intensity and detuning relative to the atomic resonance we infer $8 \times 10^{7}$ trapped atoms. This number is surprisingly large for a MOT with such a steep magnetic-field gradient. Under these conditions in conventional magneto-optical traps the Zeeman detuning rather than the size of the laser beams limits the capture volume. For cesium, a reduction in atom number by 4 orders of magnitude has been observed when the field gradient is increased from $10 \mathrm{G} / \mathrm{cm}$ to $100 \mathrm{G} / \mathrm{cm}[10]$. With the tip trap, however, we still observe $10^{5}$ trapped atoms at a field gradient of $400 \mathrm{G} / \mathrm{cm}$. We relate this effect to the field roll-off in the radial direction (fig. 2) which limits the spatial Zeeman detuning so that the magnetic field at larger distances from the center is still suitable for slowing and trapping atoms when the MOT is operated at sufficiently large laser detuning. The shape of the trapped cloud was observed with a ccd camera and may be described by a Gaussian distribution with full widths at half-maximum of $400 \mu \mathrm{m}$ in the radial and $800 \mu \mathrm{m}$ in the axial direction. This corresponds to an average number density of about $4 \times 10^{10} \mathrm{~cm}^{-3}$, which coincides with the density that was derived from the absorption of a weak probe beam.

To estimate the temperature of the atoms inside the MOT a "release and recapture" technique [11] is not applicable since at least part of the atoms will hit the needles and thus be prevented from expanding ballistically. Instead, we use an alternative method that measures the spring constant $\kappa$ of the MOT and derives the temperature from the average diameter $\langle r\rangle$ of the atom cloud [12]. Assuming the validity of the equipartition theorem the temperature is given by $k_{\mathrm{B}} T=\kappa\langle r\rangle^{2}$. To find the spring constant the atom cloud is displaced by radiation pressure of an additional laser beam which is periodically blocked by a mechanical chopper wheel at a variable frequency $\omega$. The position of the atom cloud is monitored with a 


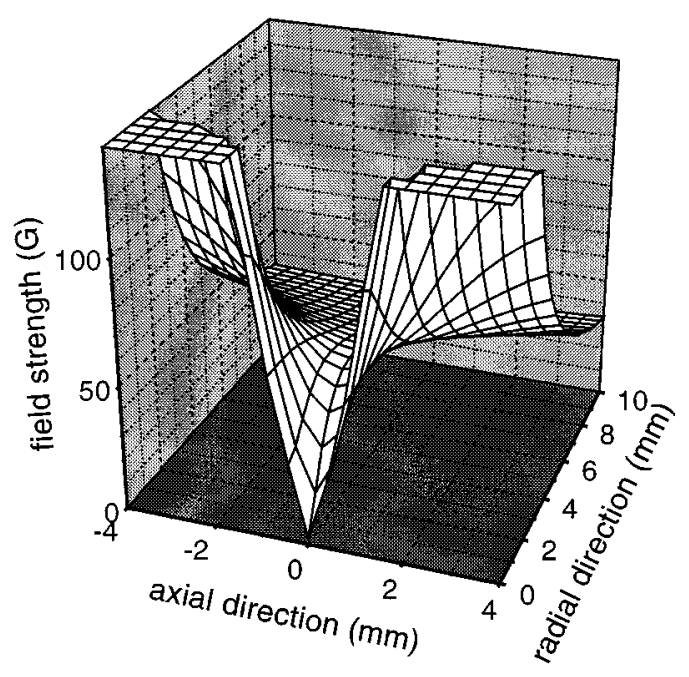

Fig. 2.

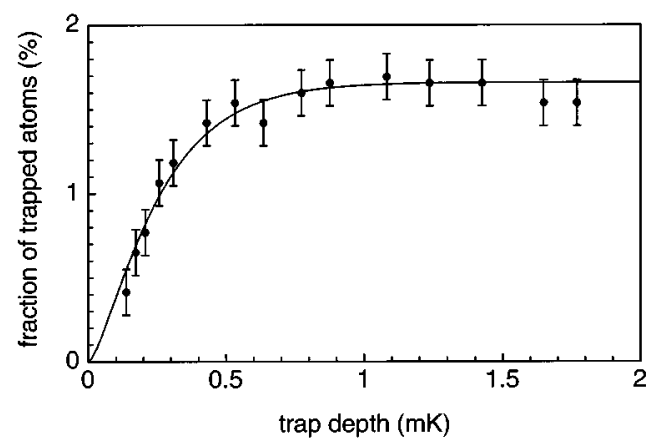

Fig. 3.

Fig. 2. - Magnetic-field strength of the configuration shown in fig. 1 for a current setting of $1 \mathrm{~A}$. The gradients in the axial and the radial direction amount to $710 \mathrm{G} / \mathrm{cm}$ and $350 \mathrm{G} / \mathrm{cm}$, respectively. At radial distances larger than $2 \mathrm{~mm}$ the field strength reaches a plateau at $50 \mathrm{G}$.

Fig. 3. - Fraction of atoms that are transferred into the purely magnetic trap. The light has been shut off for a period of $45 \mathrm{~ms}$ during which the trap depth was set to the value given by the horizontal axis. According to a simple model (solid curve) the temperature of the trapped atoms amounts to $(180 \pm 25) \mu \mathrm{K}$ assuming equal populations in the $F=2, m_{F}=1$ and $F=2, m_{F}=2$ states.

quadrant photodiode and its time phase relative to the laser light is recorded with a lock-in amplifier. By treating the atomic cloud as a driven damped harmonic oscillator the spring constant and the damping may be extracted from fitting the data to the theoretical expression $\phi(\omega)=\arctan \left(\alpha \omega / m\left(\omega_{0}^{2}-\omega^{2}\right)\right)$. The observed phases in the range between $\omega=2 \pi \times 10 \mathrm{~Hz}$ and $2 \pi \times 600 \mathrm{~Hz}$ are well described by $\alpha=3.6 \times 10^{-23} \mathrm{~kg} / \mathrm{s}$ and $\omega_{0}=2 \pi \times 335 \mathrm{~Hz}$ resulting in $\kappa=m \times \omega_{0}^{2}=5.2 \times 10^{-20} \mathrm{~kg} / \mathrm{s}^{2}$ and a temperature $T=460 \mu \mathrm{K}$. This value may be regarded only as an upper limit since the atoms are heated by the additional laser beam. Unfortunately, the oscillation cannot be induced by displacing the zero of the magnetic field because the ferromagnetic material introduces an unknown frequency-dependent phase lag between the driving current and the actual magnetic field. In fact, with a conventional MOT and a "release and recapture" method we have observed a temperature of $100 \mu \mathrm{K}$, slightly below the Doppler limit. The temperature measurement in the tip trap as discussed in the next paragraph also suggests a value very close to the Doppler limit.

The content of the MOT is transferred into the purely magnetic trap by blocking the laser with a mechanical shutter and increasing the current of the tip magnets within $200 \mu \mathrm{s}$. After a variable dark period the laser is activated again and the current set to the initial value so that all magnetically trapped atoms are rapidly transferred into the restored MOT. The resulting fluorescence signal is recorded with a photo diode and integrated for $65 \mathrm{~ms}$. The signal due to slow loading from the background gas (filling time $4 \mathrm{~s}$ ) has been carefully subtracted. Figure 3 shows the fraction of atoms that has been transferred from the initial MOT to the magnetic trap for various current settings of the electromagnets during the dark period. The number of atoms in the magnetic trap saturates for currents above $500 \mathrm{~mA}$. We interpret the data by means of a simplified model that first neglects the potential energy 
which is acquired by the atoms at the instant when the magnetic field is suddenly increased. Then, the fraction of trapped atoms is given by integrating the normalized energy distribution $N(E) \mathrm{d} E \propto E_{\mathrm{kin}}^{1 / 2} \times \exp \left[-E_{\mathrm{kin}} / k_{\mathrm{B}} T\right) \mathrm{d} E$ starting from 0 up to the trap depth $E_{\mathrm{T}}$. To estimate the influence of the sudden potential increase, one may assume that the average energy per atom is increased by the average potential energy $\langle U\rangle=-\mu b\langle r\rangle$, where $\langle r\rangle$ is the mean radius of the cloud, $b$ the magnetic-field gradient, and $\mu$ the component of the atomic magnetic momentum along the direction of the magnetic field. Since $E_{\mathrm{T}}$ is proportional to $b$ (i.e. to the current in the coils), the potential energy may be taken into account by replacing the upper integration limit $E_{\mathrm{T}}$ by $E_{\mathrm{T}}-\langle U\rangle=\alpha E_{\mathrm{T}}$. The scaling factor $\alpha:=1-\langle U\rangle / E_{\mathrm{T}}$ is estimated from the shape of the cloud as observed with a ccd camera and the calculated magnetic-field distribution to be $\alpha=0.74$. The best fit to the data is obtained for $T=(180 \pm 25) \mu \mathrm{K}$ (solid line). This suggests that despite the steep gradients the atoms may be cooled inside the MOT to a temperature close to the Doppler limit $(140 \mu \mathrm{K})$. Here we have assumed that the trapped states $F=2, m_{F}=1,2$ are equally populated. In the case that all the atoms were trapped in the $F=2, m_{F}=1\left(m_{F}=2\right)$ state the above temperature would be lower (higher) by a factor of $\sqrt{2}$. A possible population of atoms in the third trapped state, $F=1, m_{F}=-1$, has been experimentally excluded by illuminating the magnetically trapped atoms with an additional laser beam that depopulates the $F=1, m_{F}=-1$ state. No effect on the number of restored atoms has been observed. Inside the MOT the atoms tend to be pumped into the $F=2$ manifold with a preference for the states with negative magnetic quantum numbers due to the negative detuning of the laser light. This effect is more pronounced for higher gradients. The magnetically trapped states $\left(m_{F}=2,1\right)$ are thus only weakly populated, which partly explains the transfer efficiency into the magnetic trap of only $2 \%$. A transfer efficiency of $11 \%$ has been achieved by polarizing the sample immediately after the MOT has been switched off. This is accomplished by optical pumping with an additional beam for $1 \mathrm{~ms}$. The lifetime of the sample was determined by recording the number of trapped atoms after various dark periods. We observe an exponential decay with a lifetime of $7 \mathrm{~s}$. The pressure as measured with a commercial ion gauge near $(10 \mathrm{~cm})$ the trap is $3 \times 10^{-9}$ mbar. Finally, also fermionic ${ }^{6} \mathrm{Li}$ has been stored in the magnetic trap. While the transfer efficiency into the magnetic trap was similar to that of ${ }^{7} \mathrm{Li}$, ten times less atoms have been trapped inside the MOT, which may be explained by the smaller natural abundance of $7 \%$. Accordingly, the number of fermionic atoms inside the magnetic trap was $8 \times 10^{5}$.

The tip trap may be used for preparing ultra cold gases by combining it with a "top trap" that avoids the well-known Majorana transitions [6]. While the ferromagnetic needles strongly influence a static magnetic field, they have only little effect on oscillating magnetic fields at frequencies above a few $\mathrm{kHz}$. Thus, a "tip-top trap" may be realized by superimposing the oscillating field of a top trap onto the static field of a tip trap. However, the Majorana transitions inside a quadrupole trap may also be exploited to study the collision properties of the trapped gas. After a fast initial decay of atoms with small angular momentum, a further decay requires collisions that scatter atoms onto trajectories which are sensitive to Majorana flips. Since for identical fermionic lithium atoms $s$-wave scattering is forbidden, collisions are dominated by $p$-wave scattering. If, as for bosonic ${ }^{7} \mathrm{Li}, p$-wave collisions freeze out at temperatures below $1 \mathrm{mK}[13]$, the cloud of ${ }^{6} \mathrm{Li}$ is expected to be stable against Majorana transitions. In this case spin statistics changes the macroscopic properties of a cold gas even at phase space densities that are orders of magnitude below degeneracy.

$$
* * *
$$

This work was supported in parts by the Deutsche Forschungsgemeinschaft Ha 1457/4-1, the European Community HCM CT. 930105, and the German-Israeli-Foundation for scientific 
research and development I 2160167/91. We acknowledge useful discussions with L. CoLlOT, A. Hemmerich and M. Kasevich.

\section{REFERENCES}

[1] Anderson M. H., Ensher J. R., Matthews M. R., Wieman C. E. and Cornell E. A., Science, 269 (1995) 189.

[2] Davis K. B., Mewes M.-O., Andrews M. R., van Druten N. J., Durfee D. S., Kurn D. M. and Ketterle W., Phys. Rev. Lett., 75 (1995) 3969.

[3] Bradley C. C., Sackett C. A., Tollet J. J. and Hulet R. G., Phys. Rev. Lett., 75 (1995) 1687.

[4] Masuhara N., Doyle J. M., Sandberg J. C., Kleppner D. and Greytak T. J., Phys. Rev. Lett., 61 (1988) 935.

[5] Davis K. B., Mewes M.-O., Joffe M. A., Andrews M. R. and Ketterle W., Phys. Rev. Lett., 74 (1995) 5202.

[6] Petrich W., Anderson M. H., Ensher J. R. and Cornell E., Phys. Rev. Lett., 74 (1995) 3352.

[7] Abraham E. R. I., Alexander W. I., Sackett C. A. and Hulet R. G., Phys. Rev. Lett., 74 (1995) 1315.

[8] Weinstein J. D. and Libbrecht K. G., Phys. Rev. A, 52 (1995) 4004.

[9] Pritchard D. E., Raab E. L., Bagnato V., Wieman C. E. and Watts R. N., Phys. Rev. Lett., 57 (1986) 310.

[10] Höpe A., Haubrich D., Müller G., Kaenders W. G. and Meschede D., Europhys. Lett., 22 (1993) 669.

[11] Lett P. D., Phillips W. D., Rolston S. L., Tanner C. E., Watts R. N. and Westbrook C. I., J. Opt. Soc. Am. B, 6 (1989) 2084.

[12] Kohns P., Buch P., Stüptitz W., Csambal C. and Ertmer W., Europhys. Lett., 22 (1993) 517.

[13] Cote R., Dalgarno A. and Jamieson M. J., Phys. Rev. A, 50 (1994) 339. 